\title{
NOTE ON STEADY FLUID MOTION
}

\author{
BY S. D. ZELDIN
}

In a previous paper* I have shown how to find special invariant configurations of the projective and linearoid groups investigated by Wilczynski $\dagger$ in connection with steady fluid motion. It is the purpose of this note to show how the group whose general infinitesimal transformation is

$$
K f=u(x) \frac{\partial f}{\partial x}+v(x, y) \frac{\partial f}{\partial y}+w(x, y, z) \frac{\partial f}{\partial z} \ddagger
$$

should be simplified in order to represent the steady motion of a fluid under the influence of forces possessing a potential.

If the external forces have a potential, then, as is known, the functions $u, v, w$ must be such that the expression

$$
K u d x+K v d y+K w d z
$$

is a complete differential, or, what amounts to the same thing,

$$
\begin{aligned}
& \frac{\partial K u}{\partial z}-\frac{\partial K w}{\partial y}=0, \\
& \frac{\partial K w}{\partial x}-\frac{\partial K u}{\partial z}=0, \\
& \frac{\partial K u}{\partial y}-\frac{\partial K v}{\partial x}=0 .
\end{aligned}
$$

Performing the operations indicated by equations (1) we get:

$$
\frac{\partial u}{\partial x} \cdot \frac{\partial v}{\partial x}+u \frac{\partial^{2} v}{\partial x^{2}}=0
$$

(b) $\frac{\partial u}{\partial x} \cdot \frac{\partial w}{\partial x}+u \frac{\partial^{2} w}{\partial x^{2}}+v \frac{\partial^{2} w}{\partial x \partial y}+w \frac{\partial^{2} w}{\partial x \partial z}$

$$
+\frac{\partial v}{\partial x} \cdot \frac{\partial w}{\partial y}+\frac{\partial w}{\partial x} \cdot \frac{\partial w}{\partial z}=0
$$

(c) $\quad u \frac{\partial^{2} w}{\partial x \partial y}+v \frac{\partial^{2} w}{\partial y^{2}}+w \frac{\partial^{2} w}{\partial y \partial z}+\frac{\partial v}{\partial y} \cdot \frac{\partial w}{\partial y}+\frac{\partial w}{\partial y} \cdot \frac{\partial w}{\partial z}=0$,

* Journal of Mathematics and Physics (Mass. Inst. of Tech.), vol. 1 (1921), p. 54.

$\dagger$ Transactions of this Society, vol. 1 (1900), pp. 339-352.

$\$$ This class of groups has been investigated by Sophus Lie in connection with two-point invariants. See Lie-Engel, Theorie der Transformationsgruppen, vol. 3, Abtheilung 5 . 
which can be written in the form

$$
\begin{aligned}
\frac{\partial}{\partial x}\left(u \frac{\partial v}{\partial x}\right)+\frac{\partial}{\partial x}\left(v \frac{\partial v}{\partial y}\right) & =0, \\
\frac{\partial}{\partial x}\left(u \frac{\partial w}{\partial x}+v \frac{\partial w}{\partial y}+w \frac{\partial w}{\partial z}\right) & =0, \\
\frac{\partial}{\partial y}\left(u \frac{\partial w}{\partial x}+v \frac{\partial w}{\partial y}+w \frac{\partial w}{\partial z}\right) & =0 .
\end{aligned}
$$

From (a) follows at once that $K v$ can be a function of $y$ only. From $(b)$ we see that $K w$ must be a function of $y$ and $z$ alone, and, since by virtue of $(c) K w$ must be a function of $x$ and $z$ alone, it follows that $K w$ can be a function of $z$ only.

If the fluid is incompressible, we have the equation of continuity

$$
\frac{\partial u}{\partial x}+\frac{\partial v}{\partial y}+\frac{\partial w}{\partial z}=0
$$

and therefore $w$ is a linear function of $z$.

In the case of irrotational motion, since

$$
\frac{\partial w}{\partial y}-\frac{\partial v}{\partial z}=0, \quad \frac{\partial u}{\partial z}-\frac{\partial w}{\partial x}=0, \quad \frac{\partial v}{\partial x}-\frac{\partial u}{\partial y}=0,
$$

we must have, in the above infinitesimal transformation $K f$, $u$ a function of $x$ alone, $v$ a function of $y$ alone, and $w$ a function of $z$ alone. There exists then a velocity potential, say $F$, where

$$
u=\frac{\partial F}{\partial x}, \quad v=\frac{\partial F}{\partial y}, \quad w=\frac{\partial F}{\partial z},
$$

and the orthogonal trajectories of the family of surfaces

$$
F=\boldsymbol{S} u(x) d x+\boldsymbol{S} v(y) d y+\boldsymbol{S} w(z) d z=\text { constant }
$$

represent the stream lines. These stream lines are the intersections of the two families of cylinders obtained by solving the equations

$$
\frac{d x}{u(x)}=\frac{d y}{v(y)}=\frac{d z}{w(z)} .
$$

The separately invariant points will be found by solving the equations

$$
u(x)=0, \quad v(y)=0, \quad w(z)=0 .
$$

Massachusetts Institute of Technology 